\title{
Left sided arrhythmogenic ventricular dysplasia in siblings
}

\author{
C G De Pasquale, W F Heddle
}

\begin{abstract}
A 32 year old man with no previous medical history suffered a sudden cardiac death. Post mortem examination revealed circumferential fibro-fatty infiltration of the left ventricular myocardium. Histological appearance was characteristic of arrhythmogenic right ventricular dysplasia but unusual for its localisation only to the left ventricle. As a result of this sudden cardiac death the family of the deceased was screened for cardiac disease. A brother of the index case was 36 years old and free of cardiac history and symptoms. Cardiac investigations revealed a functionally and electrically abnormal left ventricle with apparent sparing of the right ventricle. The brothers may have a left sided form of arrhythmogenic ventricular dysplasia and illustrate the importance of screening family members of young victims of sudden cardiac death. (Heart 2001;86:128-130)
\end{abstract}

Keywords: left sided arrhythmogenic ventricular dysplasia

Arrhythmogenic right ventricular dysplasia (ARVD) has become established as an important cause of sudden cardiac death in the young. Although no gene has yet been identified, seven loci have been mapped in families showing autosomal dominant transmission with variable, incomplete penetrance. ${ }^{12}$

ARVD is characterised histologically by midmural or external myocardial replacement with a fibrolipomatous infiltrate..$^{2-4}$ The degree of right ventricular involvement varies from diffuse to localised infiltrations, which can give a picture of focal akinesia/dyskinesia. ${ }^{4}$

ARVD is classically described as a selective disorder of the right ventricle. Isolated case reports of left ventricular involvement in ARVD led to careful studies of left ventricular structure and function in this condition. ${ }^{56}$ These studies have shown that a significant proportion of patients have some degree of left ventricular involvement by the same disease process affecting the right. This involvement is thought to be a progression of the right ventricular disease. There are reports of apparent biventricular involvement where there is equal and extensive left and right ventricular involvement. ${ }^{3}$ The condition is not recognised as a pure left ventricular entity without right ventricular involvement. We report brothers who may represent a familial cluster of a purely left ventricular arrhythmogenic disorder bearing many similarities to ARVD.

\section{Case 1}

A 32 year old man of Anglo-Saxon background had no medical or drug taking history. He was previously asymptomatic before he collapsed while walking. Resuscitation attempts were unsuccessful. At necropsy, cardiovascular examination revealed cardiomegaly with normal coronary arteries. Macroscopic examination of the cut section of the myocardium showed a circumferential band of fibrosis measuring $1-2 \mathrm{~mm}$ in width extending between the middle and outer third of the left ventricle. The right ventricle was unremarkable (fig 1A). Histologically the left ventricle displayed patchy circumferential fibrofatty infiltration of the outer third of the myocardium (fig 1B). There was no associated inflammatory infiltrate. The right ventricle showed one small area of minor fatty infiltration with no fibrosis. There were no other necropsy abnormalities. Death was attributed to cardiac arrest with the substrate for lethal arrhythmia being ventricular dysplasia almost exclusively involving the left ventricle.

On the basis of the aforementioned pathological findings the family of the index case were screened for cardiac disease.

Family history was noteworthy for the patient's father dying a sudden cardiac death at age 48; he had suffered a myocardial infarction six years earlier. A paternal uncle with no preceding medical history also died of sudden cardiac death at age 47 . No post mortem examinations were performed on these two patients. Case 1 was the youngest of five siblings. The three oldest siblings had normal cardiac histories, examinations, and screening investigations (ECG, echocardiogram).

\section{Case 2}

Case 2 was a brother of case 1 . He was a 36 year old smoker who drank beer in moderate amounts. He had no medical history and complained of no symptoms that would arouse suspicion of cardiac disease. There were no abnormalities to be found on examination; however, his ECG showed abnormal inverted $\mathrm{T}$ waves in the inferior and lateral leads. Treadmill exercise tolerance test revealed 


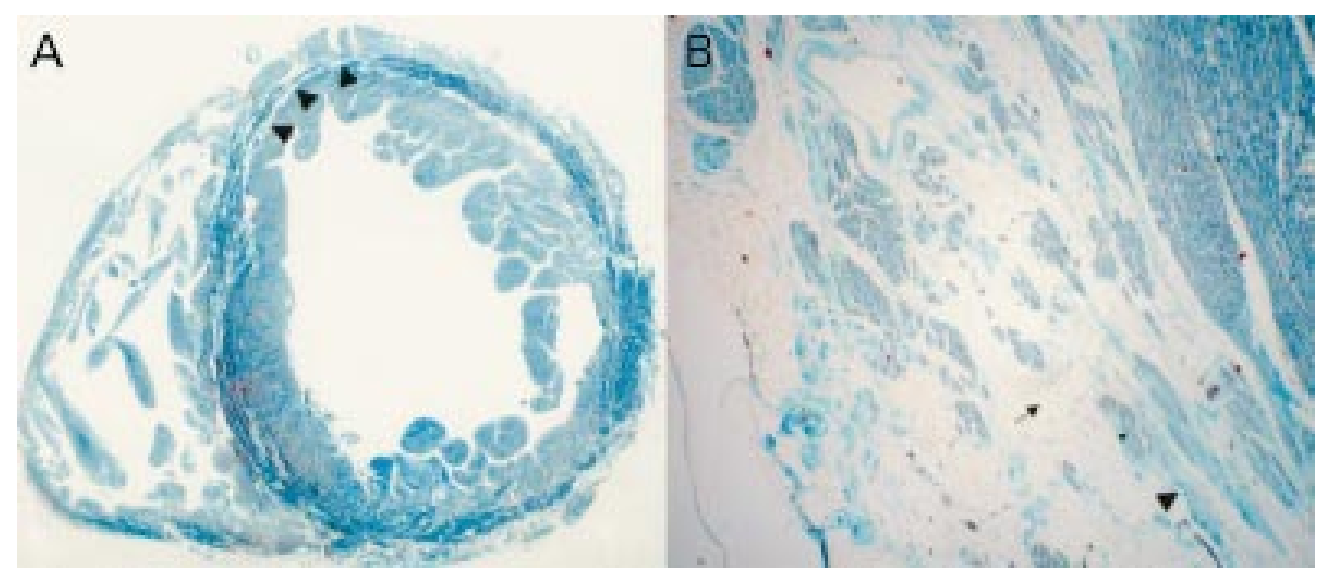

Figure 1 Case 1. (A) Left and right ventricles (trichrome stain). Cut surface showing a circumferential band of fibrosis (cyan green) (arrowheads) extending between the middle and outer third of the left ventricular myocardium (dark green) and normal appearance of the right ventricular myocardium. (B) Left ventricle (trichrome stain). Portion of left ventricle showing replacement of the outer third of the myocardium with collagenous fibrous tissue (cyan green) (large arrowhead) and adipose tissue (filigree outlining cell borders) (tailed arrow) with scattered islands of residual myocardial fibres (dark green) (small arrowhead).
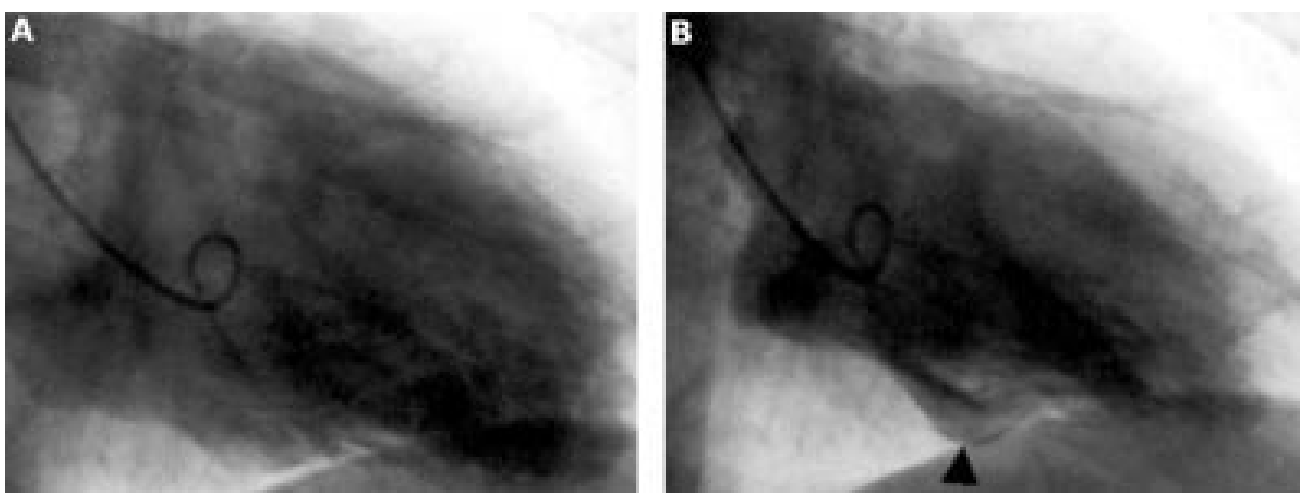

Figure 2 Case 2. Contrast ventriculography. Diastolic (A) and systolic (B) frame of left ventricular contraction showing anterior wall hypokinesis and focal dyskinetic area on inferior surface (arrowhead).

mildly reduced exercise capacity with high frequency, multifocal ventricular ectopic beats and minor ST segment depression. Echocardiography revealed a dilated left ventricle with borderline contractile function (ejection fraction $51 \%$ ); right ventricular morphology and function were normal. Signal averaged ECG was positive for late potentials and 24 hour Holter monitor revealed high frequency ventricular ectopy (25-30/1000), couplets, and an eight beat run of non-sustained ventricular tachycardia. Coronary angiography confirmed normal coronary arteries; however, left ventriculography showed mild anterior hypokinesis and focal dyskinesis in the inferior wall (fig 2A,B) Electrophysiological study revealed frequent, multifocal ventricular ectopy, couplets, triplets, and non-sustained polymorphic ventricular tachycardia throughout programmed ventricular stimulation protocol. Sustained polymorphic ventricular tachycardia requiring direct current cardioversion was initiated with three programmed extrastimuli. Cardiac magnetic resonance imaging was not performed because of technical problems with the local scanner at that time. A cardioverter defibrillator was implanted without delay. The patient was placed on an angiotensin converting enzyme inhibitor and $\beta$ blocker. At one year's follow up he had had two episodes of atrial flutter and many episodes of non-sustained VT, none of which required defibrillation.

\section{Discussion}

Diagnosis of ARVD remains difficult given the absence of an ideal diagnostic test. Many investigations reveal characteristic abnormalities but they are plagued by a lack of specificity. For this reason standardised diagnostic criteria comprising major and minor criteria have been proposed.

The changing face of the disease depending on its level of progression further complicates diagnosis. The natural history of ARVD is now considered to pass through four phases: concealed disease, overt electrical disorder, right ventricular failure, and finally biventricular pump failure. ${ }^{2}$

The cases presented in this paper fulfill several ARVD diagnostic criteria-clinically, histologically, and in their apparent inheritance. The glaring departure from ARVD is the apparent exclusive left sided nature of the disease.

Two case reports have reported entities histologically similar to ARVD with exclusively left sided disease. Collett and colleagues ${ }^{8}$ reported two cases of sudden cardiac death in young men with histopathological analysis showing extensive fibrolipomatous infiltration in the left ventricle. One subject had a completely normal 
right ventricle on histopathological examination; the other showed only one $0.5 \mathrm{~cm}$ area of fat infiltration in the right ventricular myocardium. Okabe and associates ${ }^{9}$ reported a male patient plagued for many years with ventricular arrhythmias. At necropsy cardiac examination revealed a patch of fibrolipomatous myocardial replacement not consistent with an ischaemic or inflammatory aetiology. The right ventricle was morphologically normal.

Suzuki and colleagues ${ }^{10}$ recently reported a 43 year old man who, after initially presenting with left sided cardiomyopathy with preserved right ventricular contraction, 10 years later had a histological appearance of biventricular arrhythmogenic ventricular dysplasia.

The cases presented in this report resemble those described by Collett and colleagues ${ }^{8}$ and may be noteworthy for being cases of the condition we know as ARVD localised to the left ventricle. Alternatively they may be a new genetically determined condition with similarities to ARVD being the result of the limited number of ways the heart can manifest disease.

These cases show the importance of screening the family of a young victim of sudden cardiac death as even unexpected findings may be of major importance.
We would like to thank Dr J Gilbert (Forensic Science Centre, Adelaide, Australia) for pathological details and illustrations.

1 Ahmad F, Li D, Karibe A, et al. Localization of a gene responsible for arrhythmogenic right ventricular dysplasia to chromosome 3p23. Circulation 1998;98:2791-5.

2 Corrado D, Basso C, Thiene G. Arrhythmogenic right ventricular cardiomyopathy: diagnosis, prognosis, and treatment. Heart 2000;83:588-95.

3 Fontaine G, Fontaliran F, Frank R. Arrhythmogenic right ventricular cardiomyopathies: clinical forms and main differential diagnoses. [editorial; comment] Circulation 1998;97:1532-5.

4 Marcus FI, Fontaine G. Arrhythmogenic right ventricular dysplasia/cardiomyopathy: a review. Pacing Clin Electrophysiol 1995;18:1298-314.

5 Manyari DE, Klein GJ, Gulamhusein S, et al. Arrhythmogenic right ventricular dysplasia: a generalized cardiomyopathy? Circulation 1983;68:251-7.

6 Pinamonti B, Sinagra G, Salvi A, et al. Left ventricular involvement in right ventricular dysplasia. Am Heart $\mathcal{f}$ 1992;123:711-24.

7 McKenna WJ, Thiene G, Nava A, et al. Diagnosis of arrhythmogenic right ventricular dysplasia/ cardiomyopathy. Br Heart J. 1994;71:215-8.

8 Collett BA, Davis GJ, Rohr WB. Extensive fibrofatty infiltration of the left ventricle in two cases of sudden cardiac death. F Forensic Sci. 1994;39:1182-7.

9 Okabe M, Fukuda K, Nakashima Y, et al. An isolated left ventricular lesion associated with left ventricular tachycardia. Arrhythmogenic "left" ventricular dysplasia? fpn Circ f 1995;59:49-54.

10 Suzuki H, Sumiyoshi M, Kawai S, et al. Arrhythmogenic right ventricular cardiomyopathy with an initial manifestation of severe left ventricular impairment and normal contraction of the right ventricle. Fpn Circ f 2000;64: 209-13.

\section{STAMPS IN CARDIOLOGY}

\section{Down syndrome}

Down syndrome is named after Dr John Langdon-Down (1826-1896) who was a physician at the London Hospital and was also the superintendent of the Earlswood Asylum at Redhill, Surrey where he did pioneer work in training mentally retarded children. In his Lettsom Lectures of 1887 he classified mental patients according to their facial characteristics and he labelled one as Mongolism. This term was regarded as insulting and representatives of the Mongolian Peoples Republic successfully requested the World Health Organization in 1965 to drop the term in favour of the eponym Down syndrome.

Down syndrome is caused by trisomy 21 and affects 1 in 600 neonates of whom up to $50 \%$ have congenital heart disease. The common lesions are endocardial cushion defect, ventricular and atrial septal defects, and tetralogy of Fallot, while transposition of the great arteries and coarctation of the aorta are rare.

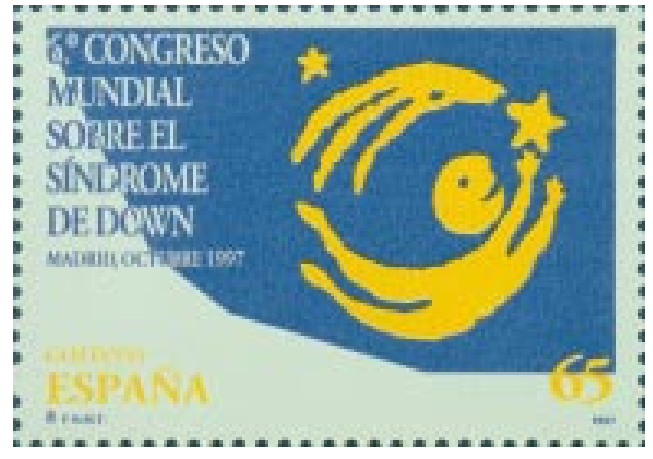

Thirty per cent of those with heart disease have multiple cardiac defects.

The 65 peseta stamp was issued by Spain to mark the Sixth World Down Syndrome Congress held in Madrid in 1997.

M K DAVIES

A HOLLMAN 